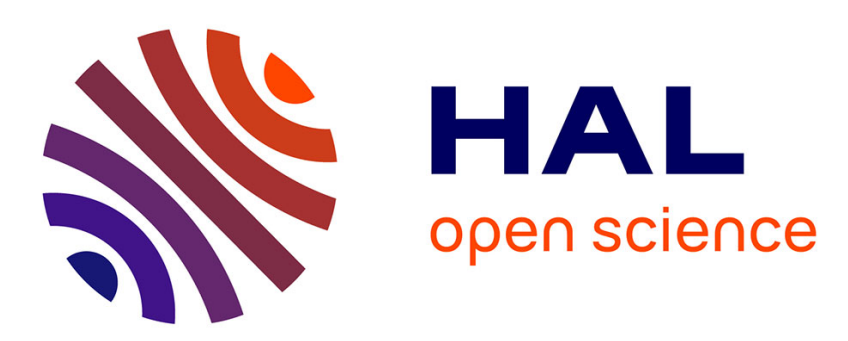

\title{
Stepwise process for the development of entecavir resistance in a chronic hepatitis $B$ virus infected patient.
}

Stéphanie Villet, Aurélie Ollivet, Christian Pichoud, Luc Barraud, Jean-Pierre Villeneuve, Christian Trépo, Fabien Zoulim

\section{To cite this version:}

Stéphanie Villet, Aurélie Ollivet, Christian Pichoud, Luc Barraud, Jean-Pierre Villeneuve, et al.. Stepwise process for the development of entecavir resistance in a chronic hepatitis B virus infected patient.: Mechanism of entecavir resistance. Journal of Hepatology, 2007, 46 (3), pp.531-8. 10.1016/j.jhep.2006.11.016 . inserm-00133115

\section{HAL Id: inserm-00133115 https://www.hal.inserm.fr/inserm-00133115}

Submitted on 23 Feb 2007

HAL is a multi-disciplinary open access archive for the deposit and dissemination of scientific research documents, whether they are published or not. The documents may come from teaching and research institutions in France or abroad, or from public or private research centers.
L'archive ouverte pluridisciplinaire HAL, est destinée au dépôt et à la diffusion de documents scientifiques de niveau recherche, publiés ou non, émanant des établissements d'enseignement et de recherche français ou étrangers, des laboratoires publics ou privés. 
Stepwise process for the development of entecavir resistance in a chronic Hepatitis B virus infected patient

Stéphanie Villet $^{1,4}$, Aurélie Ollivet ${ }^{1,4}$, Christian Pichoud ${ }^{1,4}$, Luc Barraud ${ }^{2}$, JeanPierre Villeneuve $^{3}$, Christian Trépo ${ }^{1,4,5}$, and Fabien Zoulim ${ }^{1,4,5}$

${ }^{1}$ INSERM, U271, Laboratoire des virus hépatiques et pathologies associées, Lyon, F-69003, France.

${ }^{2}$ Bioalliance Pharma, 59, boulevard Général Martial Valin, Paris, F-75015, France

${ }^{3}$ Division of hepatology, Hôpital Saint Luc du Centre Hospitalier Universitaire de Montréal, Montréal, Québec, Canada.

${ }^{4}$ Université Lyon 1, Faculté de Médecine Laennec, Lyon, F-69008, France

${ }^{5}$ Hospices civils de Lyon, Hôtel Dieu, Service d'Hépatologie, Lyon, F-69002, France

Short title: Mechanism of entecavir resistance

Contact information: Mailing address: INSERM U271, 151 cours Albert Thomas, 69424 Lyon cedex 03, France. Phone: (+33)-472681970, Fax : (+33)-472681971. Email address: zoulim@lyon.inserm.fr

Electronic word count: 3188 words 


\section{Abstract}

Background/Aims: Complex mutants may be selected under sequential anti-VHB pressures. We analyzed the genotypic and phenotypic evolution of the viral quasispecies of a patient who developed resistance to entecavir following lamivudine breakthrough.

Methods: The polymerase gene was amplified, cloned and sequenced at different time points. Hepatoma cell lines were transfected to compare the replication capacity of HBV mutants and their drug susceptibility.

Results: A mixture of lamivudine-resistant HBV strains coexisted following viral breakthrough to lamivudine, all harboring the rtM204V mutation. The rtV173L+L180M+M204V dominant mutant displayed strong lamivudine-resistance and the highest replication capacity. Following the switch to entecavir, the viral load dropped but the lamivudine-resistant strains continued to be selected. Three years later, the viral load rose again, and a complex mixture of entecavir-resistant strains, all harboring the lamivudine-resistance signature rtL180M+M204V and the rtS202G mutation were observed. Although the rtL180M+S202G+M204V variant, that prevailed at the end of entecavir therapy, did not show the highest viral genome replication capacity, it conferred one of the strongest resistance level to entecavir.

Conclusion: We report the selection of complex HBV mutants that escaped lamivudine and entecavir antiviral pressures. Genotypic and phenotypic analysis provided additional information to understand the process of HBV variant selection.

Keywords : entecavir; antiviral-resistance; HBV variants; selection process; genome evolution 
word count: 198 words 


\section{Introduction}

Hepatitis B infection affects two billion people worldwide and nearly 400 million people are chronically infected [1], resulting in severe liver disease with eventual progression to cirrhosis and hepatocellular carcinoma [2]. Alpha interferon administration has been the only treatment option for many years, and is associated with a sustained response in approximatively $30 \%$ of patients. However, dosedependent side effects are also frequent [3,4]. The development of polymerase inhibitors for the treatment of Human Immunodeficiency Virus (HIV) infection has provided a new hope for the treatment of Chronic Hepatitis B. There are currently three approved nucleos(t)ide analogs for chronic HBV infection: Lamivudine (Ldeoxycitydine analog), adefovir (adenosine monophosphate analog), and entecavir (deoxyguanosine analog). They all target the HBV reverse transcriptase (RT) activity, thus inhibiting viral replication and resulting in improvement in virologic, biochemical and histologic status in most of the patients [5-7]. However, lamivudine treatment results in the frequent emergence of drug-resistance HBV, approximatively $20 \%$ after 1 year of treatment with a progressive increase to $70 \%$ at year 4 . The main mutations associated with resistance are located in the YMDD catalytic motif of the HBV RT C domain (rtM204V/I) [8]. Compensatory mutations associated with lamivudineresistance (rtV173L, rtL180M) are found in the B domain [9,10]. The rtM204V mutation induces a 1000-fold decrease in susceptibility to lamivudine in vitro in comparison to wt HBV [11]. Adefovir-resistance occurs at a lower rate, with resistance described in $3 \%$ of patients after 2 years of treatment; this increases up to $29 \%$ by year 5 of therapy $[12,13]$. Resistance to adefovir is associated with the selection of the rtN236T mutation within the D domain of the viral enzyme [14-16] or with a rtA181V amino acid change in the B domain $[14,17,18]$. The rtN236T mutation 
results in a 3 - to 6 -fold reduction in susceptibility to adefovir in vitro $[14-16,19]$. Concerning entecavir, virologic breakthrough confirmed by genotypic analysis has been observed during phase II and III clinical trials in $5.8 \%$ of patients treated by entecavir for lamivudine failure for one year and 10\% for 2 years [20-22]. The two patients reported with resistance to entecavir had two signature lamivudineresistance mutations resulting in rtL180M and rtM204V with additional mutations of rtM250V or rtS202I. To date, phenotypic analysis revealed no de novo entecavirresistance in the absence of preexisting lamivudine-resistance [21].

In this report, we describe a new case of a patient who developed resistance to entecavir following lamivudine breakthrough. A longitudinal genotypic analysis of HBV strains isolated from the patient revealed the presence of new resistance mutations to entecavir and the persistence of lamivudine-resistance signature after three years of entecavir therapy. In vitro assays were performed to analyze the replication level and drug susceptibility of the major HBV strains. Results showed that the lamivudine-resistance signature mutations, in addition to rtS202G substitution, are necessary to induce HBV resistance to entecavir in vitro, but remain sensitive to adefovir. These results led us to gain new insight in the mechanism involved in the process of selection of entecavir-resistant mutants. 


\section{Material and Methods}

\section{Patient}

A 69 year-old Canadian man with a chronic hepatitis B and cirrhosis, was started on an Interferon therapy in May 1997. The patient was HBsAg positive and HBeAg positive. After 7 months of interferon treatment, serum HBV DNA had decreased by half. He then received lamivudine (100 mg daily). After 5 months of lamivudinetreatment, serum HBV DNA was consistently undetectable by Digene-I quantification method $\left(<1.4 \times 10^{5}\right.$ copies $\left./ \mathrm{ml}\right)$, but increased to its initial level 11 months later. He was rapidly switched to entecavir (1 mg daily). After one year of entecavir therapy, HBVDNA had decreased by three logs but viral breakthrough (more than 1-log increase) was observed two years later. The patient is currently being treated with lamivudine and adefovir combination therapy, and HBV DNA has decreased progressively by 3 logs in 2 years. The evolution of viral load over time, quantified by Digene assay (Digene, Gaithersburg, MD) during the first 84 months and by bDNA assay (Versant HBV DNA 3.0 assay; Bayer Corporation, Tarrytown, NY) the following months, is shown in figure 1. The "rebound" observed under lamivudine+adefovir bitherapy corresponds to the change in HBV DNA detection method.

\section{Clonal analysis of the HBV polymerase gene}

HBV DNA was extracted from $200 \mu \mathrm{l}$ of serum sample by using QIAamp Ultrasens virus kit according to the manufacturer's instructions (Qiagen, Hilden, Germany). The HBV polymerase gene domain between amino acid position 323 to 770 [23], encompassing the whole RT domain, was amplified by PCR using primers and thermocycling conditions described by Villet et al. [14,24]. PCR products were cloned 
into the pGEMt easy vector system (Promega, Madison, WI) according to the manufacturer's protocol. Following transformation into DH5 $\alpha$ cells (Promega), 20 colonies per serum sample were picked and the plasmid DNA inserts were sequenced.

\section{HBV constructs for phenotypic analysis}

A replication competent 1.1 HBV genome unit length vector containing HBV genome isolated from the baseline clinical sample was constructed as previously described $[14,24]$. The sequence and the replication capacity of these vectors containing HBV genome were verified.

Subsequently, the HBV polymerase mutants identified by the clonal analysis were inserted in the baseline viral genome by replacing the 1092bp Kpnl-Ncol fragment of the vector containing HBV from baseline by the corresponding KpnI-Ncol fragment from selected pGEMt-HBV polymerase plasmids. The newly obtained plasmids were verified by DNA sequencing prior to cell transfection.

\section{Construction of the rtS202G single mutant by site-directed mutagenesis}

Plasmid pGEMt containing the 1320bp wt HBV RT sequence isolated from the baseline clinical sample was engineered with the Quick Change kit (Stratagene, La Jolla, CA) to install the rtS202G mutation. The construct was sequenced to confirm this modification. The 1092bp Kpnl-Ncol fragment containing the main RT domain was then transferred to the vector containing HBV genome from the baseline clinical sample. 


\section{Analysis of HBV genome replication and drug susceptibility}

Huh-7 cells were cultured in DMEM (Eurobio, Courtaboeuf, France) supplemented with $10 \%$ fetal bovine serum. Lamivudine, tenofovir and adefovir were obtained from Gilead Sciences and entecavir from Bristol-Myers.

HBV genome replication capacity was measured by co-transfecting transiently recombinant $\mathrm{pTriex-HBV}$ clones with a luciferase reporter plasmid (pGL3, Promega) as previously described [14]. Five days after transfection, purification of HBV DNA from intracellular core particles was performed following the protocol described by Summers et al. [25]. Intracellular HBV DNA was submitted to Southern blot analysis, quantified using Phosphorlmager analysis, and adjusted for the efficiency of transfection according to the results of the luciferase assay.

The analysis of drug susceptibility was performed after transient transfection of HepG2 cells with recombinant wt and mutant pTriex-HBV clones. Sixty hours after transfection, the medium was changed and reincubated with drug-free medium or medium containing $0.01,0.1,10,100,500 \mu \mathrm{M}$ of lamivudine, or $10,20,50,100,200$ $\mathrm{nM}$ (for lamivudine-resistant clones) or 10,50, 200, 1000, $5000 \mathrm{nM}$ of entecavir (for entecavir-resistant clones), or $6.25,12.5,25,50,100 \mu \mathrm{M}$ of adefovir or tenofovir. Treatments were renewed every day for 5 days. Then, intracellular HBV DNA was purified and was subjected to Southern blot analysis as described above. For each drug, the concentration inhibiting by $50 \%$ the amount of intracellular viral DNA (IC50) detected in treated cells at the end of the treatment, compared with untreated cells, was determined by phosphorlmager analysis. 


\section{Results}

\section{Evolution of the viral RT gene in the viral quasi-species}

Viral genome analysis was performed for serum samples \#1 to \#6 throughout the clinical course of the patient (Figure 1). The DNA sequences from 20 clones per serum sample were analyzed. The sequence of the viral RT gene at baseline of lamivudine monotherapy was wild-type (wt). Five months after the beginning of lamivudine therapy, the HBV DNA titer was undetectable by Digene I assay but increased to its initial level 11 months later. At this time, surprisingly, the wt population was dominant, representing $43 \%$ of the viral population. In addition, 3 different mutants were also found to coexist: rtM204V, rtL180M+M204V and rtV173L+L180M+M204V representing 19, 9 and $29 \%$ of the viral population respectively (Figure 2). The patient was switched to entecavir and one year later, HBV DNA decreased by approximatively 3 logs. This decrease of viral load was associated with the selection of the rtV173L+L180M+M204V variant which became the major variant species representing $88 \%$ of the viral population. When viral load increased significantly 3 years after the start of entecavir therapy, the sequence analysis revealed a complex mixture of variants. All the clones maintained the lamivudine-resistance signature mutations rtL180M+M204V despite the cessation of lamivudine treatment, but also harbored the rtS202G change, not already described in the literature. The $\mathrm{rtL} 180 \mathrm{M}+\mathrm{S} 202 \mathrm{G}+\mathrm{M} 204 \mathrm{~V}$ triple mutant emerged alone or in combination with the rtl169L, rtV173L+P177S or rtA181G mutations. The triple mutant represented the major variant species, with $41 \%$ and $50 \%$ of the HBV population at months 34 and 38 of entecavir therapy respectively. More complex mutants also coexisted during this period like the rtL180M+A181G+S202G+M204V and $r$ V173L+P177S+L180M+S202G+M204V variants that represented 
approximatively $30 \%$ and $15 \%$ of the HBV population respectively. Entecavir was switched to lamivudine+adefovir bitherapy, and viral load decreased very rapidly (1 $\log$ in 1 month) and then progressively (3 logs in 2 years). Ten months after the start of lamivudine+adefovir bitherapy, we observed a more pronounced dominance of the triple mutant, and an increased proportion of the rtV173L+P177S+L180M+S202G+M204V mutant over the rtL180M+A181G+S202G+M204V variant.

\section{Viral genome replication capacity of the major HBV mutants}

To gain insight into the selection process of the different mutants, the genome replication capacity of the major HBV variants identified at each time point, was analyzed in a Huh-7 transient transfection cell culture assay as described in Material and methods (Figure 3).

We selected the 3 main mutants that emerged at the end of lamivudine monotherapy, and compared their replication capacity with that of a mixture of 10 clones, obtained from the pre-therapeutic serum, and considered as wt. The results were consistent with the data from literature, showing a higher replication capacity of the rtV173L+L180M+M204V triple mutant, compared to rtM204V and rtL180M+M204V variants (Figure 3A). Indeed, the addition of the rtL180M mutation to the rtM204V mutant induced a 1.8-fold increase of viral replication level, and the addition of rtV173L to the rtL180M+M204V a 1.7-fold increase.

The replication capacity of the four main mutants that emerged under entecavir therapy was also analyzed (Figure 3B). The last mutant selected (rtL180M+S202G+M204V) showed a slightly decreased replication capacity (71\%) compared to wt HBV. The addition of the rtA181G change to this triple mutant 
resulted in a 1.5-fold replication impairment, whereas the addition of rtl169L and rtV173L+P177S increased by 1.3- and 1.8-fold respectively the replication capacity of the same triple mutant. The rtV173L+P177S+L180M+S202G+M204V mutant even showed an increased replication efficiency (by 1.3-fold) compared to wt HBV.

\section{Drug susceptibility of the major HBV mutants}

The three main mutants previously described during lamivudine monotherapy were analyzed for their susceptibility to lamivudine and entecavir in the HepG2 cell line. Results showed that the rtL180M+M204V and rtV173L+L180M+M204V mutants were strongly resistant to lamivudine with IC50s $>10,000$-fold than that of wt HBV, and also induced a slight decrease of susceptibility to entecavir with IC50s 19-fold (rtL180M+M204V) and 30-fold (rtV173L+L180M+M204V) higher than that of wt HBV (Table 1). The rtM204V single mutant, that coexisted at low levels under lamivudine therapy and disappeared during entecavir treatment, induced only a 30- and 2.4-fold decrease in susceptibility to lamivudine and entecavir respectively (Table 1).

We also analyzed the drug susceptibility of the four main mutants that emerged during entecavir therapy. As shown in table 2, all the tested mutants were strongly resistant to lamivudine with IC50s $>500 \mu \mathrm{M}$. The triple mutant (rtL180M+S202G+M204V), which became the dominant strain at the end of entecavir and at the beginning of lamivudine+adefovir therapies, displayed with the rtl169L+L180M+S202G+M204V the highest resistance to entecavir ( 1,300- and 1200-fold compared to wt HBV). We also analyzed a mixture of the different HBV clones coexisting just before the switch from entecavir to adefovir (serum sample \#5), with the same proportion of each clone observed in serum sample \#5 by genotypic analysis. We tested the phenotype of this polyclonal mixture to adefovir and tenofovir, 
and showed the susceptibility of the mixture as well as the triple mutant to both drugs (Table 3).

\section{Implication of the rtS202G mutation in entecavir-resistance}

To determine if the rtS202G mutation alone could induce resistance to entecavir, we constructed HBV mutant by site-directed mutagenesis harboring only this mutation, and analyzed its phenotype in tissue culture. Results showed that the rtS202G mutant had a similar replication capacity and does not induce any change in entecavir susceptibility when compared to wt HBV (Table 4). Therefore, in the absence of rtL180M+M204V lamivudine-resistant substitutions, the rtS202G change is not sufficient to induce entecavir-resistance. However, the addition of rtS202G change to the rtL180M+M204V mutant resulted in a $\sim 1,300$-fold increase in entecavir-resistance. 


\section{Discussion}

This report describes virologic breakthrough after prolonged entecavir therapy in a patient who failed lamivudine therapy. A detailed clonal HBV genome analysis was performed on serum samples harvested during sequential therapies, which allowed the evolution of the viral quasi-species under the successive antiviral pressures to be shown.

Sequence analysis of HBV clones isolated from the patient's sera revealed the coexistence of 3 main populations during lamivudine therapy, all harboring the rtM204V mutation. Twenty seven months after the start of lamivudine therapy, the rtV173L+L180M+M204V triple mutant was the dominant HBV strain $(30 \%$ of HBV population). Phenotypic analysis demonstrated that this triple mutant showed the highest replicative capacity (higher than wt HBV) compared to the other lamivudineresistant variants. These results are consistent with previously published studies $[10,11,26]$. Interestingly, the triple mutant also induced a slight increase in entecavirresistance compared to the other HBV variants (30-fold versus 2.4- and 19-fold for rtM204V and rtL180M+M204V respectively). This result, in addition to a high replicative capacity, may have contributed to its selection ( $>80 \%$ of the viral quasispecies) when lamivudine was replaced by entecavir therapy. This suggests that entecavir exhibits a positive selective pressure on lamivudine-resistant mutants in vivo.

Approximatively 3 years after the beginning of entecavir therapy, we observed the coexistence of 3 or 4 mutants, all harboring the rtL180M+S202G+M204V mutations. The rtS202G change, located in the $\mathrm{C}$ domain of the $\mathrm{RT}$, has recently been observed in a patient who developed a resistance to entecavir following lamivudine breakthrough [27], in addition to lamivudine-resistance substitutions. Like the rtS202I 
mutation described by Tenney et al. [21], the rtS202G mutation has never been found alone in patients who failed entecavir therapy. This observation is consistent with our phenotypic analysis showing no decrease of entecavir susceptibility for the single rtS202G mutant, compared to wt HBV. However, the addition of rtS202G mutation to the rtL180M+M204V variant induced a $\sim 1,300$-fold decrease in susceptibility to entecavir. This result suggests that the association of the lamivudineresistance substitutions with rtS202G mutation was essential for the development of entecavir-resistance in this patient. The rtS202G change, located near the RT catalytic site, may induce a conformational perturbation of the dNTP binding pocket, preventing the binding of entecavir dGTP-TP analog [28]. This conformational change may not restore the fixation of lamivudine-TP analog, since the rtL180M+S202G+M204V mutant remained resistant to lamivudine. These data suggest that entecavir-resistance may require a two-step process with the first selection of lamivudine-resistance mutations, followed by the addition of new mutations conferring a higher replication capacity in the presence of entecavir. It is important to note that this triple mutant remains sensitive to adefovir and tenofovir in vitro (Table 3). This susceptibility to adefovir was confirmed in vivo with the decrease of viral load following a switch to adefovir.

New mutations were also found in some HBV clones from this patient in association with rtL180M+S202G+M204V mutations. The rtA181G substitution, was present in 30 to $40 \%$ of the HBV population at the end of entecavir therapy, but the proportion of this mutation decreased when entecavir was replaced by lamivudine+adefovir. This alanine in 181 position seems to be critical in the development of resistance to nucleos(t)ide analogs, since i) it is located in $\alpha$-helix adjacent to the nucleotide binding site [29], and ii) rtA181V and rtA181T were already described to confer 
resistance to lamivudine and adefovir [28]. Interestingly, phenotypic assays demonstrated that the rtL180M+A181G+S202G+M204V mutant does not reduce susceptibility to adefovir (data not shown), and has a slightly lower replication capacity when compared to the triple mutant, which may explain the decrease of proportion of this mutant under lamivudine+adefovir treatment.

The rtP177S mutation was always found in association with rtV173L in addition to rtL180M+S202G+M204V changes in the patient, except for one clone (data not shown). This mutant harboring five closed mutations in RT protein represented approximatively $15 \%$ of the population at the end of entecavir therapy, and its proportion slightly increased after the switch from entecavir to lamivudine+adefovir. A mutation at this position in leucine, but not serine, has already been described for resistance to famciclovir [30]. This proline at the 177 position is conserved in HIV RT (P157), and seems to directly interact with M184 in HIV RT or M204 in HBV RT [28]. This substitution, like the mutation at the 181 position, could alter the positioning of the nucleic acid template strand relative to the catalytic region. In our phenotypic analysis, we found that rtP177S, in association with rtV173L, did not increase resistance of the triple mutant to entecavir, but induced a 1.8-fold increase in replication capacity of the same mutant. Since rtV173L was already described to support replication of the rtL180M+M204V lamivudine-resistant mutant, it would be interesting to study the implication of each mutation rtV173L or rtP177S in this phenomenon.

A third new mutation, rtl169L, was also found in a few HBV clones isolated from the patient, but only at one time point (34 months after the start of entecavir therapy). The mutation of this position was already described in the two patients described by Tenney et al., who developed entecavir resistance, but threonine and not leucine 
substituted the isoleucine [21]. This rtl169L seems to induce a modest increase in the triple mutant replication level (1.3-fold), but did not increase entecavir-resistance level.

When we compared the phenotype of these mutants coexisting during entecavir therapy in our tissue culture assay, we observed that the rtL180M+S202G+M204V triple mutant that was dominant during entecavir therapy, conferred one of the highest resistance to entecavir in vitro ( 1,300-fold). This mutant did not show the highest replication level, but its replication capacity was only slightly impaired compared to wt HBV (by approximatively $30 \%$ ). Therefore, we can hypothesize that entecavir pressure selected the viral mutant that was the best fit in the presence of entecavir.

In conclusion, we report a case of resistance to entecavir following lamivudine breakthrough. Viral breakthrough was associated with the emergence of the entecavir-resistance substitution rtS202G, in addition to lamivudine-resistant mutations. There is currently no observation of entecavir-resistance in the absence of previous lamivudine treatment failure. The longitudinal genotypic analysis of HBV clones, combined with the in vitro phenotypic analysis of the major HBV variants, allows insight into the understanding of the selection process of the entecavirresistant mutants, which followed a two-step model in our patient. Our findings highlight the importance of in vitro cross resistance testing, since adefovir was shown to inhibit in vitro the replication of these entecavir-resistant and reduced the viral load after the development of entecavir-resistance. 


\section{Acknowledgements}

This work was supported by grants from the European Community and part of the activities of the ViRgil network of excellence (ViRgil LSHM-CT-2004-503359). 


\section{References}

[1] Lee WM. Hepatitis B virus infection. N. Engl. J. Med. 1997;337:1733-45.

[2] Lavanchy D. Hepatitis B virus epidemiology, disease burden, treatment, and current and emerging prevention and control measures. J Viral Hepat 2004;11 (2):97-107.

[3] Lau GK, Piratvisuth T, Luo KX, Marcellin P, Thongsawat S, Cooksley G, Gane E, Fried MW, Chow WC, Paik SW, Chang WY, Berg T, Flisiak R, McCloud P, Pluck N. Peginterferon Alfa-2a, lamivudine, and the combination for HBeAgpositive chronic hepatitis B. N Engl J Med 2005;352 (26):2682-95.

[4] Wong DK, Cheung AM, O'Rourke K, Naylor CD, Detsky AS, Heathcote J. Effect of alpha-interferon treatment in patients with hepatitis $B$ e antigenpositive chronic hepatitis B. A meta-analysis [see comments]. Ann Intern Med 1993;119 (4):312-23.

[5] Marcellin P, Chang TT, Lim SG, Tong MJ, Sievert W, Shiffman ML, Jeffers L, Goodman Z, Wulfsohn MS, Xiong S, Fry J, Brosgart CL. Adefovir dipivoxil for the treatment of hepatitis $B$ e antigen-positive chronic hepatitis $B$. N Engl $J$ Med 2003;348 (9):808-16.

[6] Leung NW, Lai CL, Chang TT, Guan R, Lee CM, Ng KY, Lim SG, Wu PC, Dent JC, Edmundson S, Condreay LD, Chien RN. Extended lamivudine treatment in patients with chronic hepatitis $B$ enhances hepatitis $B$ e antigen seroconversion rates: results after 3 years of therapy. Hepatology 2001;33 (6):1527-32.

[7] Chang TT, Gish RG, de Man R, Gadano A, Sollano J, Chao YC, Lok AS, Han KH, Goodman Z, Zhu J, Cross A, DeHertogh D, Wilber R, Colonno R, Apelian 
D. A comparison of entecavir and lamivudine for $\mathrm{HBeAg}$-positive chronic hepatitis B. N Engl J Med 2006;354 (10):1001-10.

[8] Lai CL, Dienstag J, Schiff E, Leung NW, Atkins M, Hunt C, Brown N, Woessner M, Boehme R, Condreay L. Prevalence and clinical correlates of YMDD variants during lamivudine therapy for patients with chronic hepatitis $B$. Clin Infect Dis 2003;36 (6):687-96.

[9] Melegari M, Scaglioni PP, Wands JR. Hepatitis B virus mutants associated with 3TC and famciclovir administration are replication defective. Hepatology 1998;27:628-33.

[10] Delaney WEt, Yang H, Westland CE, Das K, Arnold E, Gibbs CS, Miller MD, Xiong S. The hepatitis B virus polymerase mutation rtV173L is selected during lamivudine therapy and enhances viral replication in vitro. J Virol 2003;77 (21):11833-41.

[11] Allen MI, Deslauriers M, Andrews CW, Tipples GA, Walters KA, Tyrell DLJ, Brown N, Condreay LD. Identification and characterization of mutations in hepatitis B virus resistant to Lamivudine. Hepatology 1998;27:1670-7.

[12] Hadziyannis SJ, Tassopoulos NC, Heathcote EJ, Chang TT, Kitis G, Rizzetto M, Marcellin P, Lim SG, Goodman Z, Ma J, Arterburn S, Xiong S, Currie G, Brosgart CL. Long-term therapy with adefovir dipivoxil for HBeAg-negative chronic hepatitis B. N Engl J Med 2005;352 (26):2673-81.

[13] Locarnini S QX, Arterburn S. Incidence and predictors of emergence of adefovir resistant HBV during four years of adefovir dipivoxil (ADV) therapy for patients with chronic hepatitis B (CHB). 40th Annual Meeting of the European Association for the Study of the Liver. Paris, France, 2005. 
[14] Villet S, Pichoud C, Villeneuve JP, Trepo C, Zoulim F. Selection of a multiple drug-resistant hepatitis B virus strain in a liver-transplanted patient. Gastroenterology 2006;131 (4):1253-61.

[15] Villeneuve JP, Durantel D, Durantel S, Westland C, Xiong S, Brosgart CL, Gibbs CS, Parvaz P, Werle B, Trepo C, Zoulim F. Selection of a hepatitis B virus strain resistant to adefovir in a liver transplantation patient. J Hepatol 2003;39 (6):1085-9.

[16] Angus P, Vaughan R, Xiong S, Yang H, Delaney W, Gibbs C, Brosgart C, Colledge D, Edwards R, Ayres A, Bartholomeusz A, Locarnini S. Resistance to adefovir dipivoxil therapy associated with the selection of a novel mutation in the HBV polymerase. Gastroenterology 2003;125 (2):292-7.

[17] Fung SK, Andreone P, Han SH, Rajender Reddy K, Regev A, Keeffe EB, Hussain M, Cursaro C, Richtmyer P, Marrero JA, Lok AS. Adefovir-resistant hepatitis B can be associated with viral rebound and hepatic decompensation. J Hepatol 2005;43 (6):937-43.

[18] Osiowy C, Villeneuve JP, Heathcote EJ, Giles E, Borlang J. Detection of rtN236T and $\mathrm{rtA} 181 \mathrm{~V} / \mathrm{T}$ mutations associated with resistance to adefovir dipivoxil in samples from patients with chronic hepatitis B virus infection by the INNO-LiPA HBV DR line probe assay (version 2). J Clin Microbiol 2006;44 (6):1994-7.

[19] Brunelle MN, Jacquard AC, Pichoud C, Durantel D, Carrouee-Durantel S, Villeneuve JP, Trepo C, Zoulim F. Susceptibility to antivirals of a human HBV strain with mutations conferring resistance to both lamivudine and adefovir. Hepatology 2005;41 (6):1391-8. 
[20] Sherman M, Yurdaydin C, Sollano J, Silva M, Liaw YF, Cianciara J, BoronKaczmarska A, Martin P, Goodman Z, Colonno R, Cross A, Denisky G, Kreter B, Hindes R. Entecavir for treatment of lamivudine-refractory, HBeAg-positive chronic hepatitis B. Gastroenterology 2006;130 (7):2039-49.

[21] Tenney DJ, Levine SM, Rose RE, Walsh AW, Weinheimer SP, Discotto L, Plym M, Pokornowski K, Yu CF, Angus P, Ayres A, Bartholomeusz A, Sievert W, Thompson G, Warner N, Locarnini S, Colonno RJ. Clinical emergence of entecavir-resistant hepatitis B virus requires additional substitutions in virus already resistant to Lamivudine. Antimicrob Agents Chemother 2004;48 (9):3498-507.

[22] Chang TT, Gish RG, Hadziyannis SJ, Cianciara J, Rizzetto M, Schiff ER, Pastore G, Bacon BR, Poynard T, Joshi S, Klesczewski KS, Thiry A, Rose RE, Colonno RJ, Hindes RG. A dose-ranging study of the efficacy and tolerability of entecavir in Lamivudine-refractory chronic hepatitis B patients. Gastroenterology 2005;129 (4):1198-209.

[23] Stuyver LJ, Locarnini SA, Lok A, Richman DD, Carman WF, Dienstag JL, Schinazi RF. Nomenclature for antiviral-resistant human hepatitis B virus mutations in the polymerase region. Hepatology 2001;33 (3):751-7.

[24] Durantel D, Carrouee-Durantel S, Werle-Lapostolle B, Brunelle MN, Pichoud C, Trepo C, Zoulim F. A new strategy for studying in vitro the drug susceptibility of clinical isolates of human hepatitis B virus. Hepatology 2004;40 (4):855-64.

[25] Summers J, Smith PM, Horwich AL. Hepadnaviral envelope proteins regulate covalently closed circular DNA amplification. J. Virol. 1990;64:2819-24. 
[26] Seigneres B, Aguesse-Germon S, Pichoud C, Vuillermoz I, Jamard C, Trepo C, Zoulim F. Duck hepatitis B virus polymerase gene mutants associated with resistance to lamivudine have a decreased replication capacity in vitro and in vivo. J Hepatol 2001;34 (1):114-22.

[27] Yim HJ, Hussain M, Liu Y, Wong SN, Fung SK, Lok AS. Evolution of multidrug resistant hepatitis B virus during sequential therapy. Hepatology 2006;44 (3):703-12.

[28] Bartholomeusz A, Tehan BG, Chalmers DK. Comparisons of the HBV and HIV polymerase, and antiviral resistance mutations. Antivir Ther 2004;9 (2):149-60.

[29] Das K, Xiong X, Yang H, Westland CE, Gibbs CS, Sarafianos SG, Arnold E. Molecular modeling and biochemical characterization reveal the mechanism of hepatitis B virus polymerase resistance to lamivudine (3TC) and emtricitabine (FTC). J Virol 2001;75 (10):4771-9.

[30] Xiong X, Yang H, Westland CE, Zou R, Gibbs CS. In vitro evaluation of hepatitis $B$ virus polymerase mutations associated with famciclovir resistance. Hepatology 2000;31 (1):219-24. 


\section{Figure legends}

Figure 1. Evolution of the viral load during the course of antiviral therapies. The duration of therapies is indicated by bars above the graph and the serum samples from the patient by the numbers below the graph. Lamivudine, entecavir, and adefovir dipivoxil were administred to the patient. The evolution of viral load over time was quantified by Digene assay (Digene, Gaithersburg, MD) during the first 84 months and by bDNA assay (Versant HBV DNA 3.0 assay; Bayer Corporation, Tarrytown, NY) the following months. The rebound observed under lamivudine+adefovir bitherapy corresponds to the change in HBV DNA detection method.

Figure 2. Genotypic analysis of HBV mutants evolution during sequential therapies. The different bars correspond to the percentage of each HBV RT mutant in the viral population. On the left are indicated the serum sample numbers.

Figure 3. Replicative capacity in Huh-7 cells of wt and HBV RT mutants isolated from the patient during lamivudine (A) and entecavir therapy (B). Viral DNA synthesis was analyzed as described in Material and Methods. After phosphorimager analysis, values obtained for each mutant were divided by wt HBV value that was assumed to represent $100 \%$ of viral replication. Values are reported as the mean and standard deviation from six independent experiments. 